\title{
INTENSITY VARIATIONS IN THE SOLAR EXTREME ULTRAVIOLET SPECTRUM
}

\section{OBSERVED BY OSO-1}

\author{
By Werner M. NeUpert \\ (Goddard Space Flight Center, Greenbelt, Maryland U.S.A.)
}

\begin{abstract}
RAsumt. - On passe en revue les observations de l'extrême ultraviolet solaire obtenues en mars, avril et mai 1962 par le satellite $O S O$. On discute l'emploi de ces résultats pour l'identification des raies d'émission dans le domaine spectral 170-400 §. Ces observations sont comparées à celles du flux radioélectrique sur $2800 \mathrm{MHz}$ et à d'autres mesures de l'activité solaire.
\end{abstract}

ABSTRACT. - This paper reviews solar extreme ultraviolet observations made in the spectral region $\lambda 170-\lambda 400$ by the first Orbiting Sclar Observatory during March, April, and May, 1962. Application of the data to the identification of emission lines in this spectral region is discussed. The observations are compared with concurrent $2800 \mathrm{MHz}$ radio emission and other measurements of solar activity.

Резюме. - Просматрены наблюдения в краиней ультрафиолетовой солнечной области, полученные в марте, апреле и мае 1962Г спутником OSO. Обсуждено применение этих результатов для отождествления эмиссионных линий в спектральной области 170-400 А̊. Эти наблюдения сравнены с наблюдениями радиоэлектрического потока в 2800 Мгц и с другими измерениями солнечной активности.

\section{INTRODUCTION.}

This paper reviews the solar extreme ultraviolet (EUV) observations made by the first Orbiting Solar Observatory, OSO-1, using a grazing incidence spectrometer. The experiment was performed by the Solar Physics Branch of the Goddard Space Flight Center, under the direction of Dr. John C. LINDSAY and Dr. William E. BEHRING. As an experiment in the pointed section of the OSO-1 spacecraft, the spectrometer made continuous solar observations, whenever the satellite was in sunlight, for nearly three solar rotations in March, April, and May 1962. These observations were followed by nearly one year of intermittent observations. During the period of continuous observations EUV spectra were obtained at the rate of one every eight minutes, or approximately seven per orbit, resulting in an estimated seven thousand spectra being recorded for the first 2 1/2 months of satellite operation. Reports of these observations may be found in the literature [1], [2], [3].

This paper will discuss observations made on some of the more prominent spectral lines in the region from $\lambda 170$ to $\lambda 400$ made during March, April, and May 1962. In particular, the discussion will center around :
1) The use of satellite observations in the identification of spectral lines.

2) The use of these observations to study changes in the solar corona with time, especially those changes associated with active centers and flares.

\section{INSTRUMENTATION.}

During operation the spectrometer [4] was pointed at the center of the solar disk with an accuracy of two arc minutes. In this orientation, radiation from the entire solar disk and inner corona passed directly through the entrance slit and struck a concave grating mounted in grazing incidence, the angle of incidence being $88^{\circ}$. The grating, an original ruled in a special glassby the Nobel Institute in Stockholm, has 576 lines per millimeter on a blank of one meter radius of curvature. The exit slit and detector were mounted on a carriage which was driven on a circular rail so that the exir slit scanned along the Rowland Circle, where the spectrum was focused, from $\lambda 10-\lambda 400$. The 50 micron entrance and exit slits provided a spectral passband of $1.7 \AA$ and permitted resolution of lines $0.85 \AA$ apart. The detector was a windowless photomultiplier developed by the Bendix Corporation specifically for use 
in this spectrometer. A tungsten photocathode was chosen to minimize response to wavelengths above $\lambda 1500$, and to reduce changes in sensitivity due to variations of the emission properties of the cathode. For the OSO-1 flight spectrometer, calibrations were performed (1) by exposing the entire spectrometer to a beam of monochromatic radiation of known intensity and (2) by evaluating the essential components of the spectrometer (grating, detector, etc...) and then computing the sensitivity of the instrument. The first method was applied at $\lambda 44$ using a proportional counter for determining the source intensity. The second method was applied at longer wavelengths $(\lambda 80-\lambda 400)$. In addition, a comparison was made with the fluxes measured by Hall, Damon and Hinteregger [5] on a day of equivalent solar activity as measured by the solar decimetric radiation. The comparison could only be made in the region of overlap, $\lambda 250$ to $\lambda 400$, and would be meaningful only if the solar EUV raoiation was indeed the same on the two days. The $2800 \mathrm{MHz}$ mean daily flux recorded by the National Research Council, Ottawa. Canada, was used as an independent estimate of solar flux to choose the satellite data for the comparison. A best fit was made between these methods of obtaining a calibration which yielded values of $8.0 \times 10^{5}$ photons $\mathrm{cm}^{-2}$ count $^{-1}$ at $\lambda 335$ and $4.2 \times 10^{5}$ photon $\mathrm{cm}^{-2}$ count $^{-1}$ at $\lambda 284$.

The first use of this spectrometer, in a rocket flight on September 30,1961 prior to launch of the OSO-1 satellite, produced the spectrum shown in Figure 1. The most reliable observations were obtained in the region from $\lambda 170$ to $\lambda 400$ and agree well with observations made in this same region by HaLl et al. [6] at Air Force Cambridge Research Laboratories and TouSEY et al. [7] at the Naval Research Laboratory. Among the more prominent features of the spectrum are the Helium II sequence with lines at $\lambda 304, \lambda 256$ and $\lambda 243$, and the Fe XV and Fe XVI resonance lines at $\lambda 284$ and $\lambda 335$ respectively. By combining several scans, it was possible to obtain evidence for line structure down to $\lambda 50$. Both the NRL and AFCRL groups recently have obtained superior spectra extending below $\lambda 100$.

The type of data provided during operation of the satellite is shown is Figure 2. These data were taken on two days of the more than two months of continuous observations made by OSO-1 and show the effects of changing solar activity. It is immediately evident that the
Fe XVI ( $\lambda 335)$ and Fe XV ( $\lambda 284)$ lines increase relatively more than do neighbouring lines as the activity on the Sun increases between March 9 and March 22. An alternative way of looking at the data is to consider one line only, but over a longer period of time. One again concludes that the fluctuations due to solar activity may differ considerably from one line to another. These fluctuations have been investigated with regard to information concerning line identification and coronal structures which may be obtained from them.

\section{Identification OF SPECTRAL LINES.}

The problem of identifying coronal emission lines has been discussed by Tousey, Austin, Purcell and Widing [7], by Zirin, Hall and Hinteregaer [8], by Pecker and Rohrlich [9], and Neupert and Behring [10]. One of the difficulties lies in the nature of the far UV spectrum. It is a rich emission line spectrum which prevents one from making unambiguous identifications when the predicted wavelengths are good to only a few Angstroms. We suggest that this ambiguity can be removed by making observations over a period of time, and searching for groups of lines whose intensities have the same time dependence. Assuming that the lines are optically thin, and that the excitation cros-section is only a slowly varying function of temperature, we may expect that lines of one multiplet arising from one or several closely spaced upper states will vary by the same fractional amount with changes in solar activity. This imposes an additional constraint which line identifications must satisfy.

This method has been used in a search for the permitted transitions of Fe XIV in the solar spectrum (NEUPERT and SMITH [11]). Predictions for the energies of the excited levels of this ion have been made by Garstang [12] and allow one to calculate the expected wavelengths with reasonable accuracy. One expects from GarsTANG'S calculations that the EUV lines of this ion are grouped into four multiplets appearing in the extreme ultraviolet spectrum between $\lambda 200$ and $\lambda 400$. It is evident that the problem of identification is simplified by knowing precisely the splitting of two lines having a common upper state, since this splitting must correspond to the wave number of the green line at $\lambda .5303$. Upon examining the time variations of lines in the region of the spectrum where Fe XIV is expected 

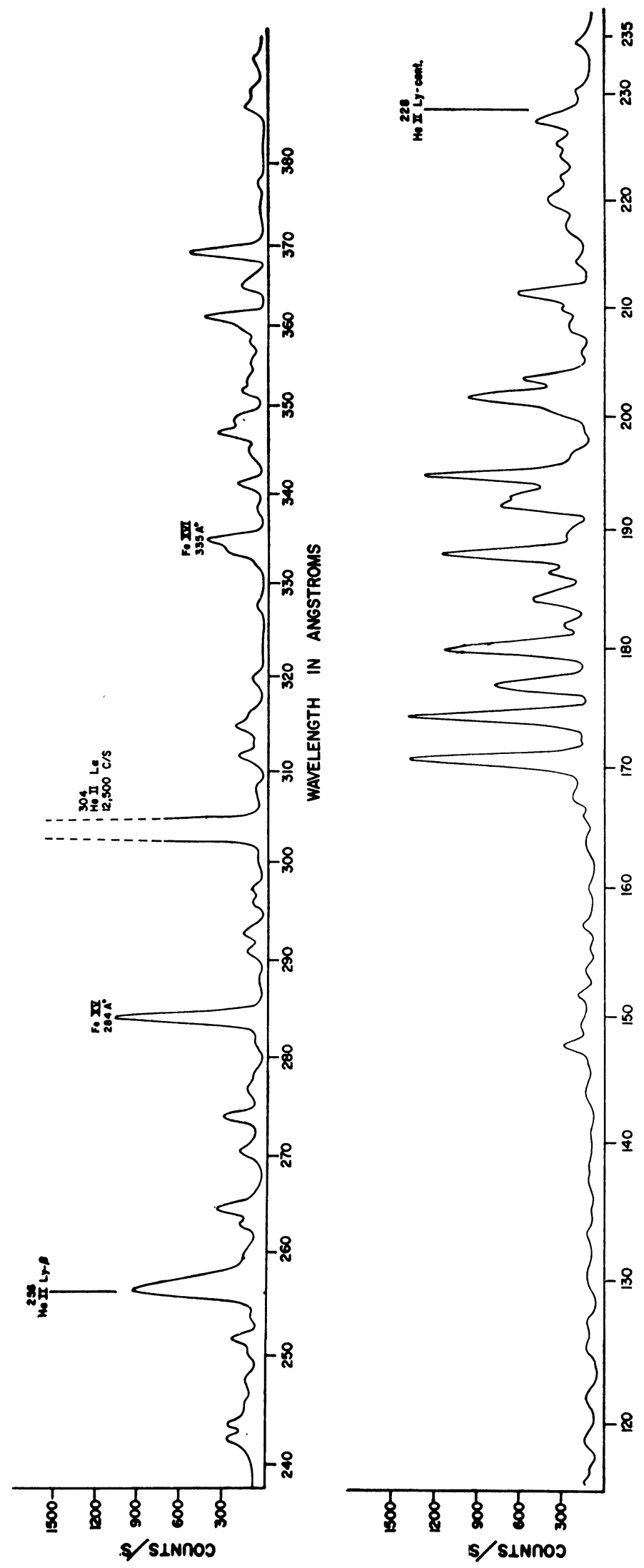

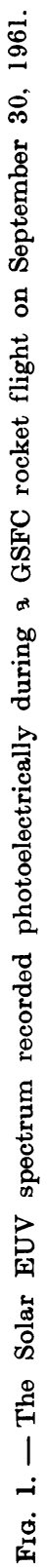
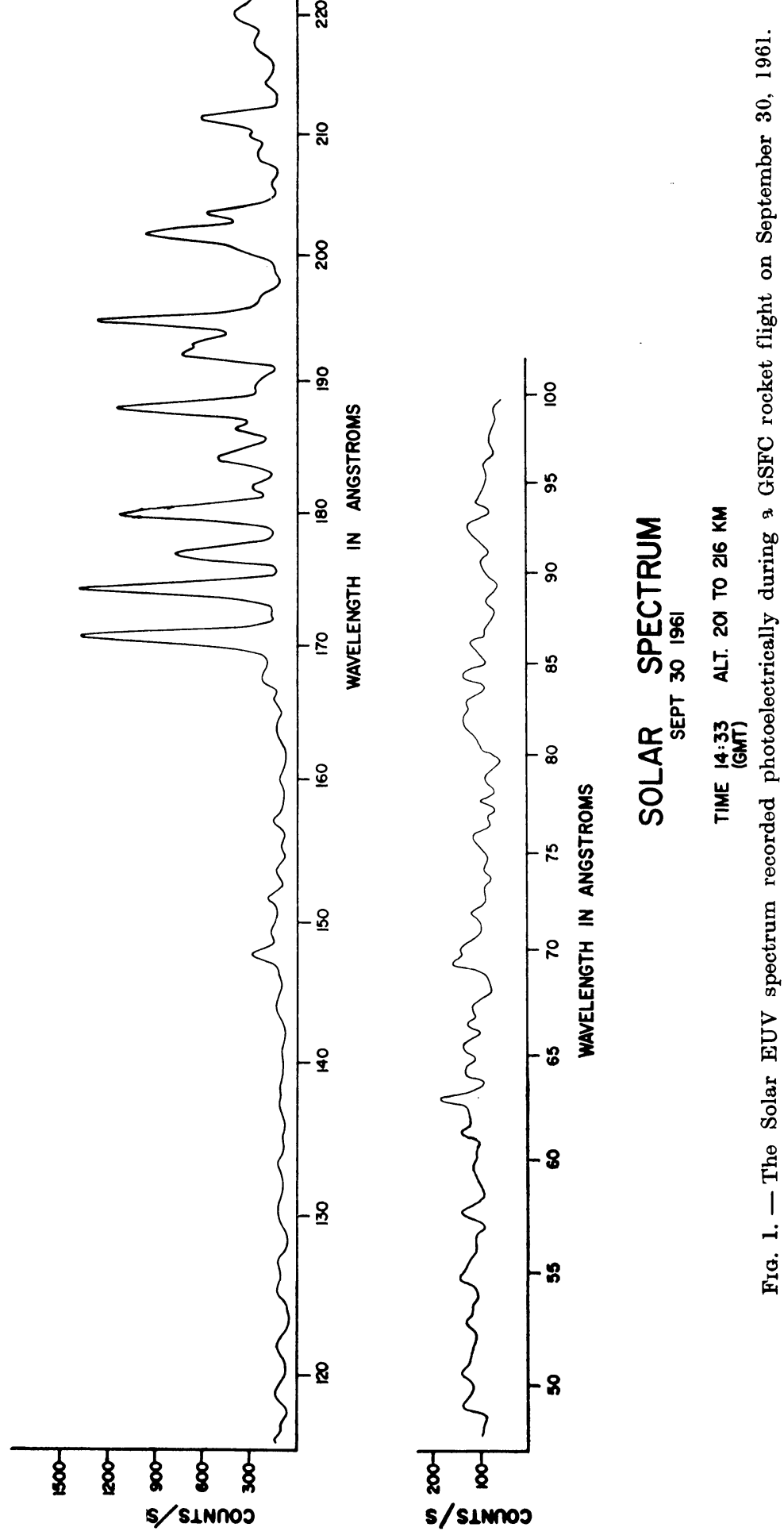


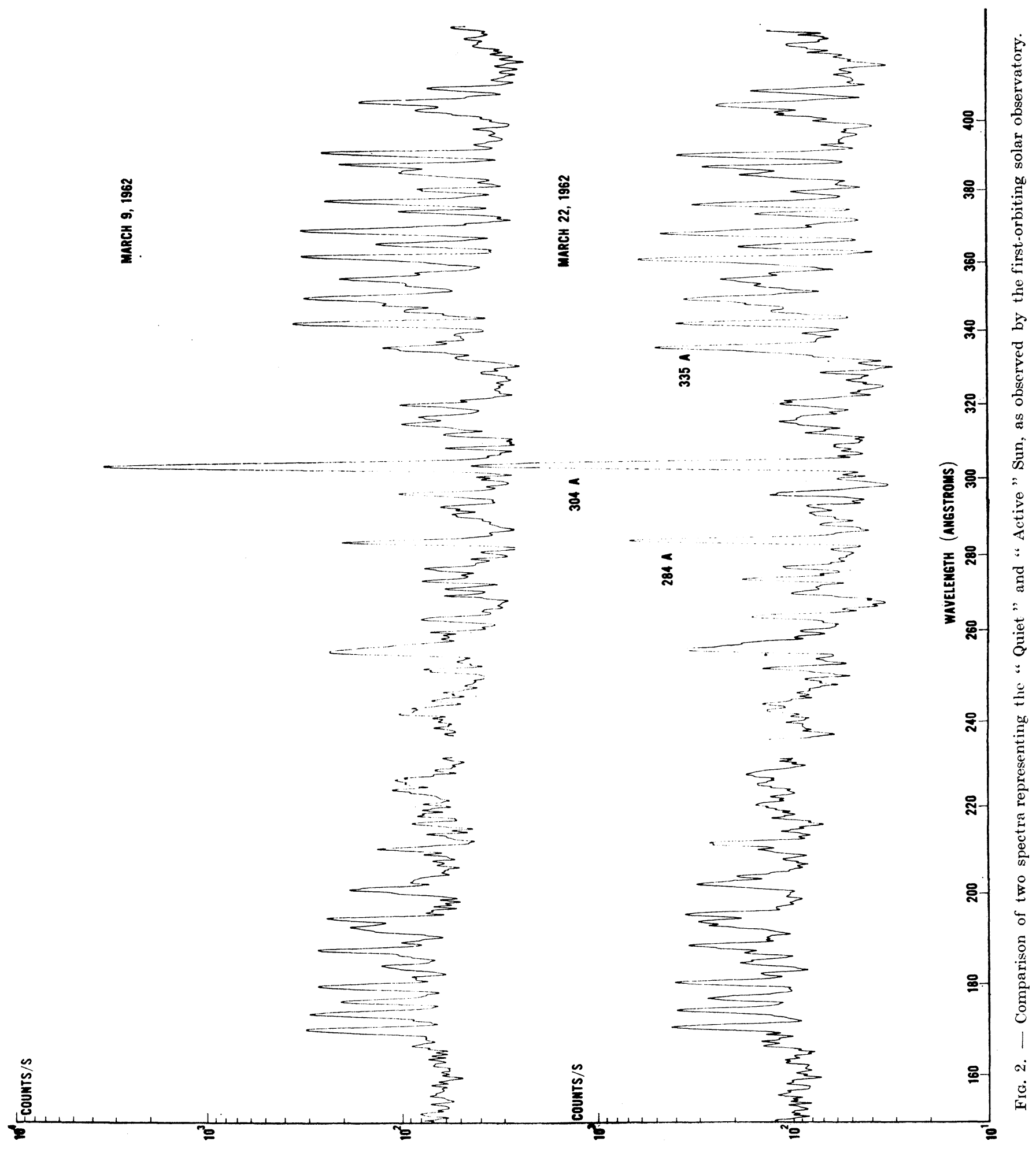


one does find three groups of lines with wavelengths near those calculated from GarsTaNa's work, and with separations compatible with predicted splittings within the uncertainty in determining the wavelengths of the observed lines, which vary by the same amount as the activity on the Sun changes. We suggest that two of these groups correspond to the ${ }^{2} \mathrm{P}-{ }^{2} \mathrm{Po}$ and ${ }^{2} \mathrm{~S}-{ }^{2} \mathrm{P}^{0}$ transitions since they lie within $2 \AA$ of the computed wavelengths. Two other lines, at $\lambda 204 \mathrm{~A}$ and $\lambda 211$ have the same variation with time and probably correspond to the ${ }^{2} \mathrm{D}-{ }^{2} \mathrm{Po}^{0}$ transitions although they are shifted by $10 \AA$ from the predicted wavelengths. None of the other lines we have examined exhibit the same time dependence. The resonance lines, expected above $\lambda 340$, are not observed. Their absence may be due to their rather low oscillator strengths, as computed by Garstang, or to the decreased sensitivity of the OSO-1 spectrometer at these wavelengths. The similarity in intensity fluctuations exists for the entire period of observation. The collected observations are shown in Figure 3, in which each group of points corresponds to one multiplet. There is good agreement not only among members of the same multiplet but also from one multiplet to another. The $\lambda 289$ line appears to deviate, but this is a weak line and may be blended with other lines, especially during periods of low solar activity. Table I summarizes the possible identifications.

Having examined one set of lines which may originate in iron, it is of interest to examine other

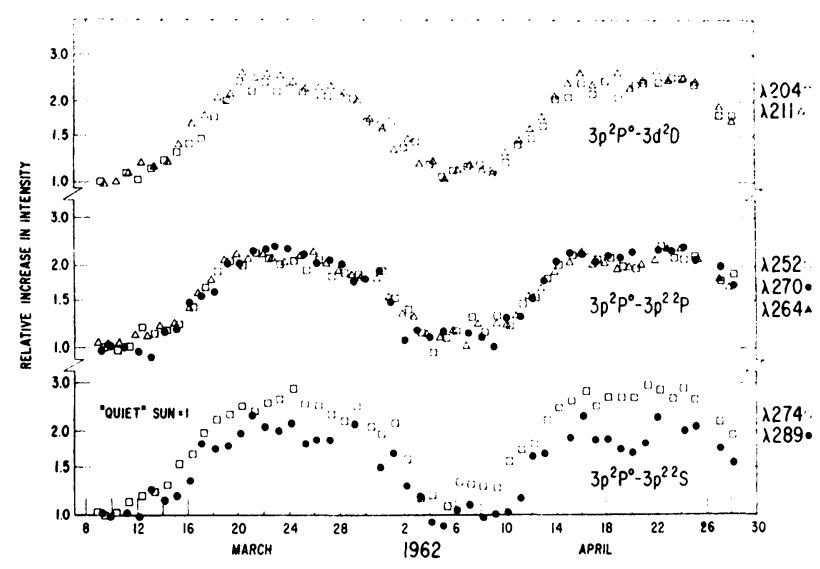

Frg. 3. - Intensity fluctuations of possible Fe XIV EUV emission lines. The lines are normalized to a value of one for a period of time during which the Sun was considered as "Quiet" for this portion of the solar cycle.

lines of the same element which perhaps belong to different stages of ionization. To do this, one may turn to those lines which are common to both the Zeta discharge and the Sun and which, as FAWCETT, et al. [13] have pointed out, may be iron lines. The intensity variations of those lines which are reliably observed by the OSO-1 spectrometer are shown in Figure 4. These lines have been divided into four groups according to the amplitude of the fluctuations. No other lines show as much variation as do the lines of Group IV which we have suggested as being from Fe XIV, but lesser variations are observed. Of these lines, one group, Group III, may originate

TABLE I

Predicted and observed Fe XIV lines in the solar euv.

\begin{tabular}{|c|c|c|c|c|c|}
\hline Transition & $\mathrm{J}_{\mathrm{L}}-\mathrm{J}_{\mathrm{U}}$ & $\begin{array}{c}\text { Predicted } \\
\text { WAVELENGTH } \\
\text { (GARSTANG) }\end{array}$ & $\begin{array}{c}\text { OBSERVED } \\
\text { WAVELENGTH }{ }^{1} \text { ) }\end{array}$ & $\begin{array}{c}\text { Wavenumber } \\
\text { Computed } \\
\text { (GARSTANG) }\end{array}$ & $\begin{array}{l}\text { OBSERVED }\left({ }^{1}\right. \\
\text { DIFFERENCES }\end{array}$ \\
\hline - & - & - & - & - & - \\
\hline $3 p^{2} \mathrm{Po}-3 p^{2}{ }^{2} \mathrm{~S}$ & $\begin{array}{l}1 / 2-1 / 2 \\
3 / 2-1 / 2\end{array}$ & $\begin{array}{l}272.9 \\
287.7\end{array}$ & $\begin{array}{l}274.3 \\
288.7\end{array}$ & 18860 & 18180 \\
\hline $3 p^{2} \mathrm{P}^{\circ}-3 p^{2}{ }^{2} \mathrm{P}$ & $\begin{array}{l}0 \\
1 / 2-1 / 2 \\
1 / 2-3 / 2\end{array}$ & $\begin{array}{l}256.0 \\
250.5\end{array}$ & $\begin{array}{l}\text { Blend } \\
251.8\end{array}$ & & \\
\hline & $\begin{array}{l}3 / 2-3 / 2 \\
3 / 2-1 / 2\end{array}$ & $\begin{array}{l}262.9 \\
269.0\end{array}$ & $\begin{array}{l}264.5 \\
270.5\end{array}$ & $\begin{array}{r}18860 \\
8640\end{array}$ & $\begin{array}{r}19070 \\
8380\end{array}$ \\
\hline $3 p^{2} \mathrm{P}^{0}-3 d^{2} \mathrm{D}$ & $\begin{array}{l}1 / 2-3 / 2 \\
3 / 2-3 / 2 \\
3 / 2-5 / 2\end{array}$ & $\begin{array}{l}214.3 \\
223.1 \\
222.0\end{array}$ & $\begin{array}{l}203.9 \\
211.5\left({ }^{2}\right)\end{array}$ & $\left.\begin{array}{l}18860 \\
16536\end{array}\right\}$ & $17620\left(^{2}\right)$ \\
\hline
\end{tabular}


in Fe XIII, as two suggested identifications by ZIRIN [14], and the similarity of the variations with those of another possible Fe XIII line at $\lambda 364$ would suggest. The remaining lines have been placed into two categories but these categories are not distinct. The least variation is

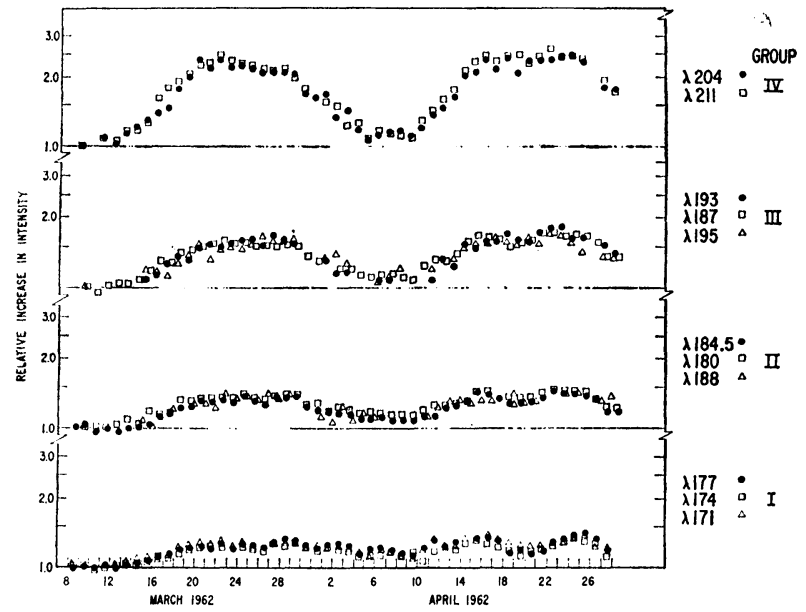

FIG. 4. - Intensity fluctuations of emission lines common to both the Zeta Pinch and the Sun. Only those lines which have been reliably observed by OSO-l are included. The same normalization is used as in Figure 3.

shown, not only by a group of lines near $\lambda 170$, but also by a possible $\mathrm{Fe} \mathrm{X}$ line at $\lambda 345$ and leads us to suggest that these lower two groups originate in $\mathrm{Fe} \mathrm{X}, \mathrm{Fe} \mathrm{XI}$, and Fe XII, the smallest variation being associated with $\mathrm{Fe} \mathrm{X}$. Figure 5 shows the corresponding spectra from Zeta and TousEY's spectrum of the Sun [13], and again indicates the tentative grouping and identification of the spectral lines.

In addition to the iron lines, we have examined variations observed in silicon and magnesium lines. Rather than present data for each ion in detail, one may attempt to summarize these observations from another point of view. We find that, in general, those ions which exist at lower electron temperatures vary less than those which exist at higher temperature. This behaviour is shown in Figure 6 . The temperatures used here are those given by House [15] and do not include the effect of dielectronic recombination. Including this factor will shift the points and may change the shape of the dotted curve slightly. This dotted curve varies from day to day, of course, depending on solar activity. We shall refer to this figure again after discussing the solar activity with which the observations are associated.

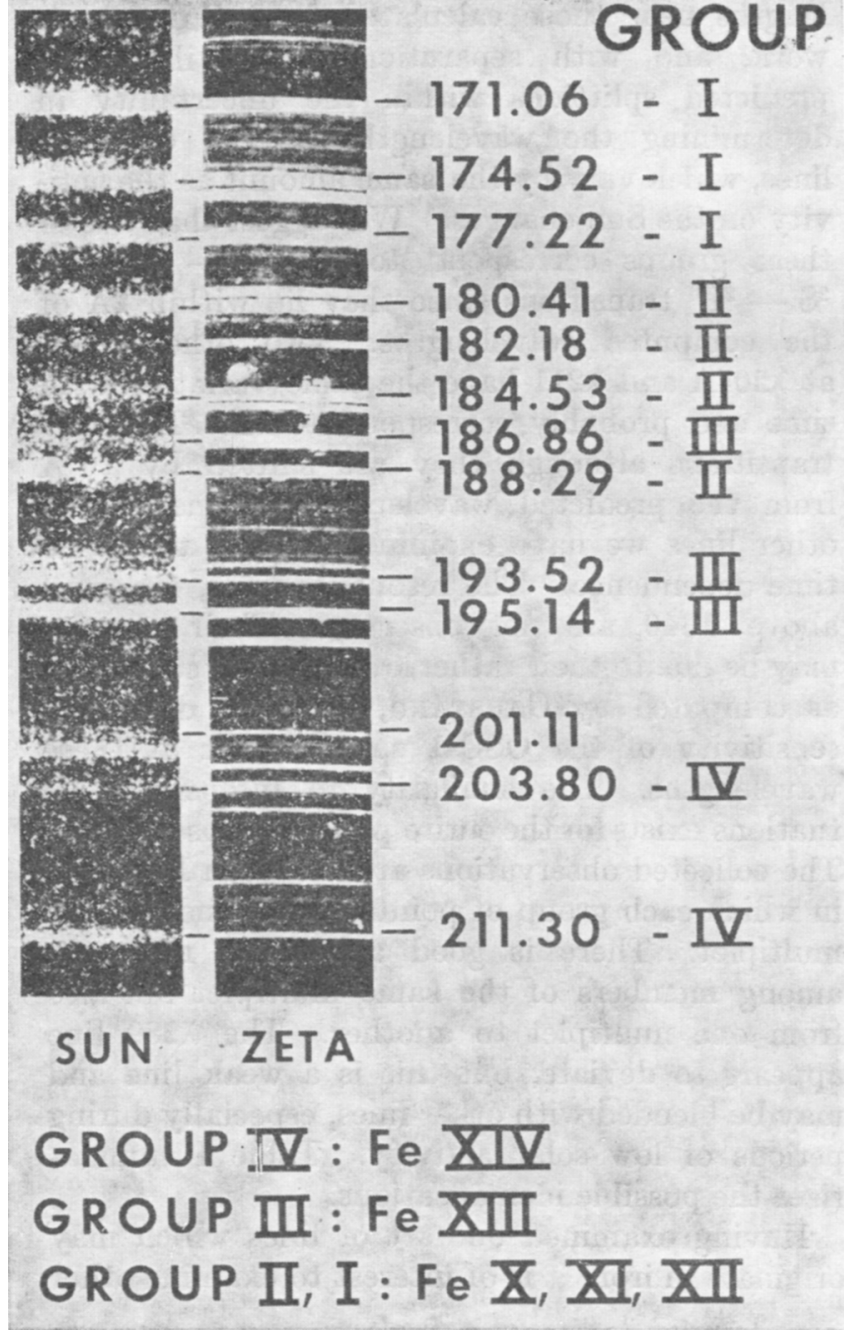

Frg. 5. - Comparison of Solar and Zeta Spectra (Fawcett et al $^{13}$ ) Showing the suggested identifications of some of the brighter lines. (Spectra reproduced with the kind permission of the authors.)

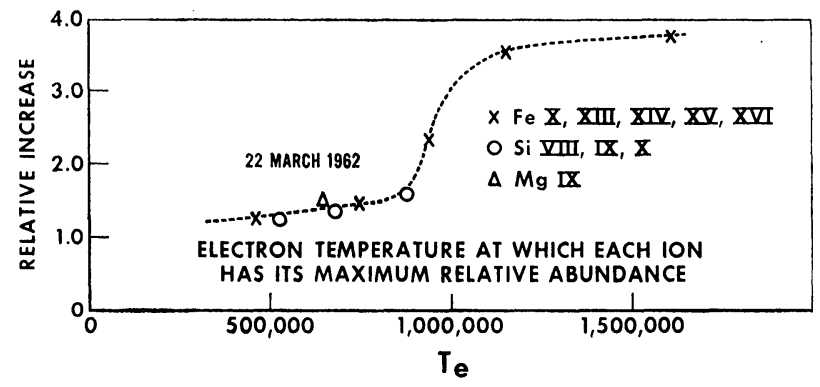

Fig. 6. - Increases in EUV emission lines due to the presence of active centers (March 22, 1962) relative to a period of low solar activity (March 10, 1962). The temperatures used in this Figure do not take into account the effect of dielectronic recombination. 


\section{VARIATIONS OF THE EUV SPECTRUM WITH SOLAR ACTIVITY.}

The use of the Orbiting Solar Observatory as a stable platform has permitted the acquisition of a solar EUV spectrum which can tentatively be associated with a corona disturbed to varying degrees by vibible centers of activity. Figure 7 shows the fortuitous sequence of observations obtained by OSO-1: periods of relatively low solar activity followed by periods during which active centers appeared on the solar disk. The increases and decreases in flux can be associated with the appearance and dissappearance of these centers, as indicated by the plage regions given in the figure. The elosest approach to observarion of a corona not disturbed by active centers was obtained on $11 \mathrm{March}$, when the Zurich Final Relative Sunspot Number was 11. The American Relative Sunspot Number was reported as zero on both 10 March and 11 March. In addition, no large regions had been associated with the face of the Sun turned toward the earth on

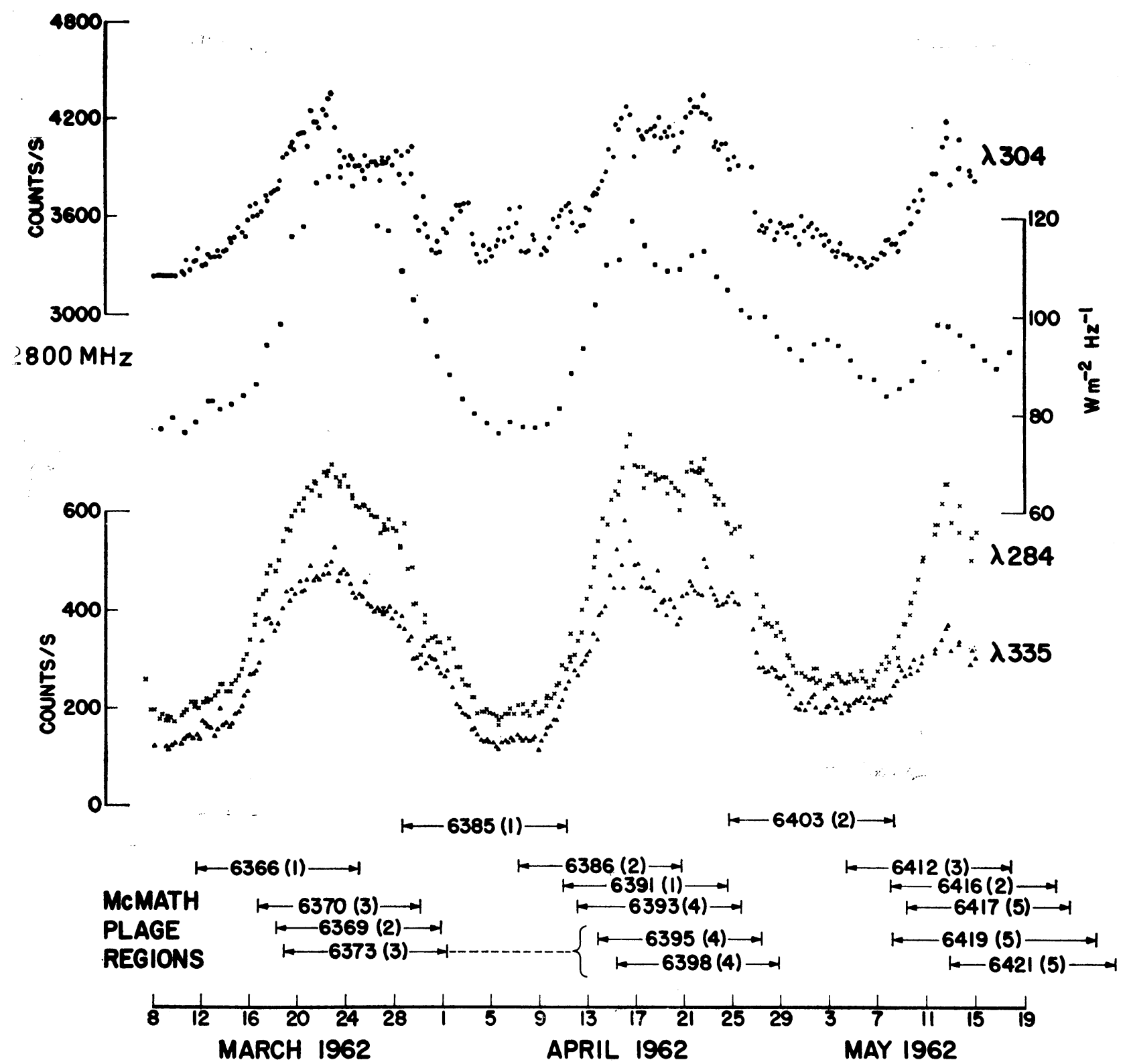

Fia. 7. - Comparison of three selected EUV emission lines with mean daily $2800 \mathrm{MHz}$ radio emission reported by the National Research Council, Ottawa, Canada and Ca+ Plages reported by the McMATH-HunBirrt Observatory. Age of each plage, in solar rotations, is given within parenthesis. 
March 11 during the preceding six months. A careful analysis of emission lines, made for the period from 7 March to 5 April, demonstrates that the lowest counting rates of the period were indeed observed when the sunspot number was near zero and the calcium plage area on the Sun was also at a minimum. However, it is also clear that no exact correlation can be assumed to exist between the EUV fluxes and ground-based observations. For example, counting rates for the iron lines were lower on May 1, when the Sunspot Number was 49 than on May 15 when it was 15. Likewise, although the agreement between the radio data obtained at $2800 \mathrm{MHz}$ by the National Research Council, Ottawa, Canada, and EUV corcnal fluxes is striking at times, as for instance in the interval from 13 April to 25 April, a period of considerable solar activity, this similarity is not consistent, as the data taken from 29 April to 15 May shows. A small maximum observed at $2800 \mathrm{MHz}$ on 1 May to 5 May does not appear at $\lambda 284$ whereas on the succeeding days, 9 May to 15 May, a larger peak is observed at $\lambda 284$ than at radio wavelengths. The region under observation on 1 May to 5 May when a small maximum is observed at $2800 \mathrm{MHz}$ is a flare-producing center of activity approximately 1 solar rotation old, whereas the plages existing on the Sun on 9 May to 15 May are for the most part remnants of active centers 4-5 rotations old which no longer are sites of flare activity.

The EUV observation would suggest that the $\lambda 284$ radiation has a different time dependence on the age of active regions than does the microwave radiation. It increases more slowly than does the microwave radiation as the active center develops but remains intense even after the sunspots and flare activity have dissappeared and the microwave radiation is decreasing. One may compare this hypothesis with a model for a center of activity described by De JAGER [16]. In this model the disturbance is initially localized in the lower altitudes of the solar atmosphere and gradually expands into the quiet corona. After the spot phase of the CA has passed, the center remains as a magnetic bipolar region which may have quiescent filaments and perhaps coronal rays associated with it. The continued enhancement of the Fe XV line after all sunspots have vanished in the region may be an indication of remaining coronal structures. In any event these observations suggest that it is necessary to have knowledge of the recent past history of solar activity as well as current data in order to make a correlation of EUV radiation with other data. The three EUV emission lines for which data are given display individual features which vary from one line to another. In particuar, one may observe fluctuations in the helium line which are not found in the other lines. These short lived variations can sometimes, but not always, be associated with the brightening of existing plages and the occurrence of radio noise storms at $169 \mathrm{MHz}$ as recorded by the Meudon Field Station in Nançay, France.

The coronal lines of Fe XIV, Fe XV, and Fe XVI are strongly associated with plages, but do appear to have residual intensities even if the Sun shows no sign of activity. A more detailed analysis for $\mathrm{Fe} X \mathrm{XV}$ and for Fe XIV appears in Figure 8. One

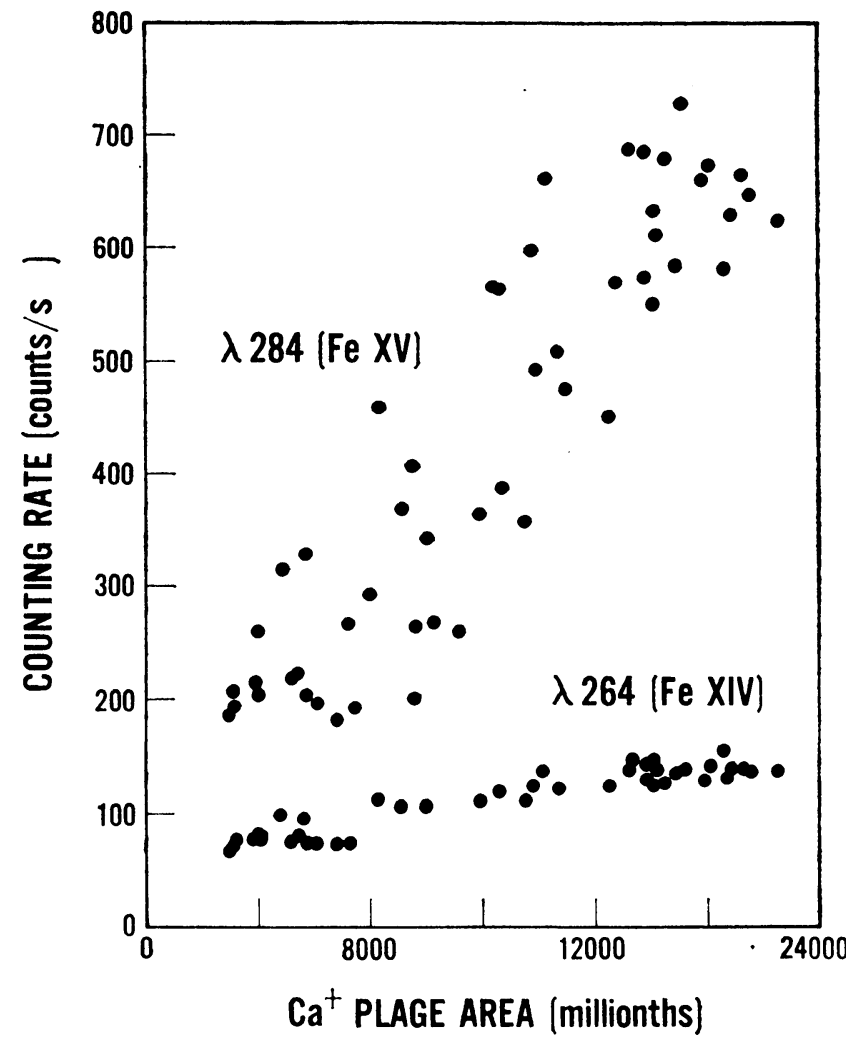

Fra. 8. - Correlation of Fe XV and Fe XIV Lines with Ca+ Plage Area as Observed by the McMath-Hulbert Observatory.

may observe that although there are large fluctuations in the relationship of Fe XV to plage area, the Fe XV emission is more strongly associated with plages than Fe XIV. A " quiet Sun" component, of about 90 counts per second does exists when one extrapolates the $\mathrm{Fe} \mathrm{XV}$ counting rate to zero plage area. Assuming that the regions of increased $\mathrm{Fe} \mathrm{XV}$ emissions are equivalent in area to the plages, one may calculate a plage to quiet, 
Sun Fe XV ratio of between two and three hundred to one, considerably beyond the latitude of photographic film. This " quiet Sun" component may perhaps be associated with coronal fine structure rather than be uniformly distributed over the solar disk. It may be that with sufficient sensitivity and spatial resolution we will find a mottled appearance to the solar disk even in the lines of $\mathrm{Fe} \mathrm{XV}$ and Fe XVI. If one assumes that both of these radiations originate in the same regions of the corona, at some well-defined electron temperature, one obtains, from the theory of ionization equilibrium, a temperature of the order of 1.75 million degrees. This is to be compared with the value of 800,000 oK usually obtained for undisturbed regions of the corona in which the forbidden lines of $\mathrm{Fe} \mathrm{XIV}$ and $\mathrm{Fe} \mathrm{X}$ have an intensity ratio of about four to one.

It is of interest to compare the Fe XVI and XV radiations associated with successive reappearances of the same active regions. This comparison is presented as the ratio of counting rates observed for the $\lambda 335$ and $\lambda 284$ lines and is shown in Figure 9. It is immediately evident that this ratio is insensitive to the occurrence of major active regions. It appears, however, that a slightly higher ratio may be associated with the above mentioned month-old region observed from 1 Mav to 5 May and that a somewhat lower ratio exists from 12 May to 15 May, when primarily regions approximately 5 months old were under observation. This ratio also decreases slightly with each successive reappearance of the major active regions which made central meridian passage on March 23-25.

The data suggest that the Fe XV emission from the latter regions remains approximately the same throughout the period of observarion while a decrease in Fe XVI occurs at about the same time that flare activity ceases and sunspots vanish. If the regions of Fe XV and XVI emissions were to coincide, the observations would imply that a higher electron temperature is to be associated with the younger region. There is, however, a small amount of evidence in the data that the two radiations do not always precisely coincide. On 17-19 March, 30 March-2 April, and 11-13 April we observe increases in the ratio as a major plage area, which is increasing in activity, is situated behind either the east or west limb of the Sun.

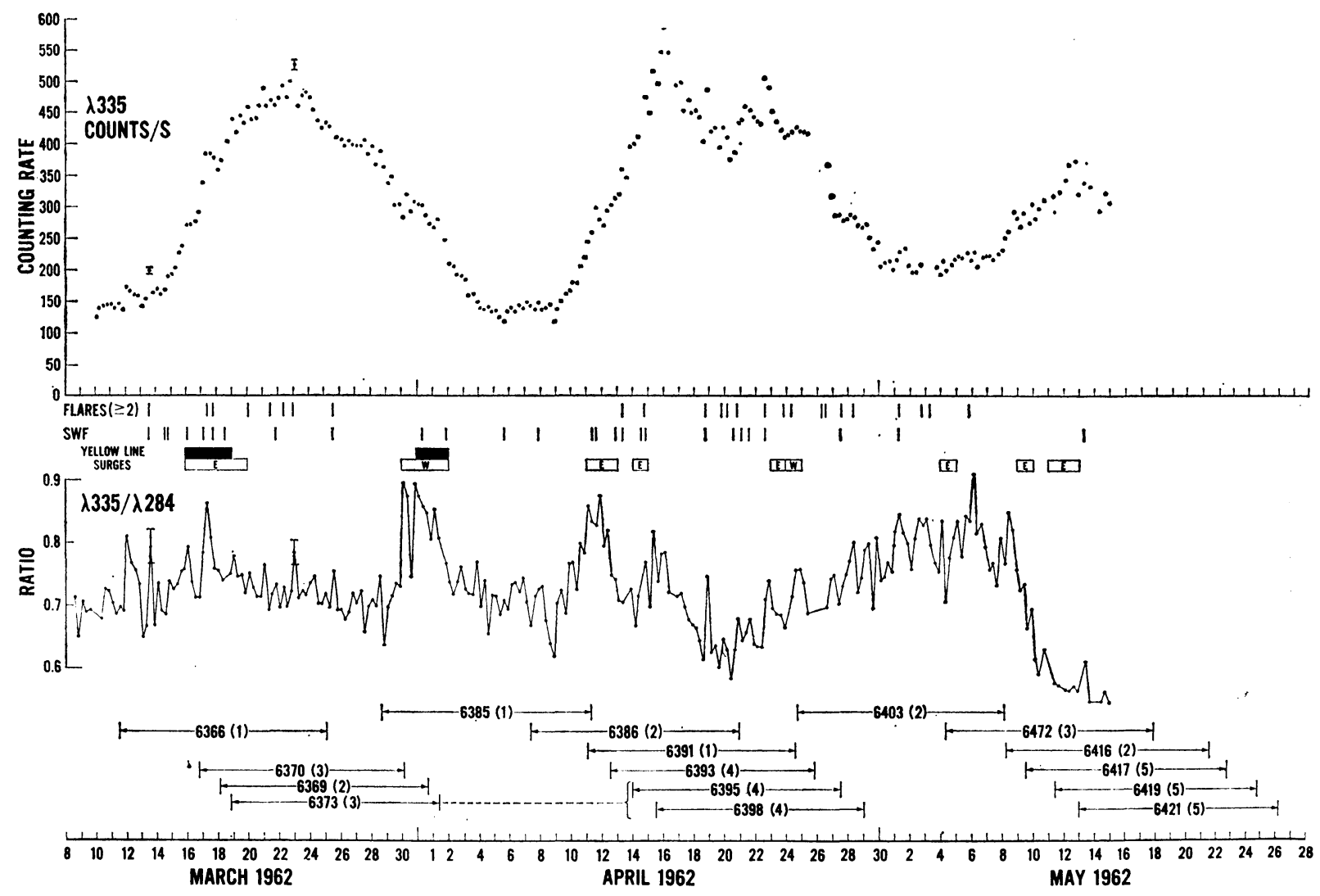

FIG. 9. - Dependence of the ratio Fe XVI/Fe XV on solar activity. occurrence of flares of importance 2 or greater, short-wave fadeouts, and limb activity is also shown. 
Such an increase would be obtained if the Fe XVI emission occurred somewhat higher in the corona than did the $\mathrm{Fe} X V$, a situation which could be produced by an electron temperature gradient above the plage. Several different coronal models are now being investigated in this regard.

Finally, referring back to Figs. 3, 4, and 6, we may attempt to summarize the fluctuations observed in other EUV lines as a result of activity on the Sun. We observe that those ions which exist at electron temperatures below about $1 \times 10^{6} \mathrm{oK}$, and these include the lower stages of ionization of iron as well as ions of Si VIII through Si X and Mg VIII and IX, show little association with active regions, while those ions existing above $1 \times 10^{6} \mathrm{oK}$ show a strong if not exclusive association with plages and active regions. The fact that all lines show an increase with activity during the first two weeks in March merely indicates an increase in density in and around the active region. It is not clear from the data whether the large increases in $\mathrm{Fe} X V$ and $\mathrm{Fe}$ XVI are due to a combined increase of electron temperature and density over plages or whether localized regions in which these emissions might occur merely increase in number over plages. All lines are observed to fluctuate, if only slightly. The smaller the fluctuations, the less well they correlate with solar activity. This phenomenon is particularly well demonstrated in Figure 4. Since the experiment lacked spatial resolution it is not possible to state how the smaller enhancements in intensity are distributed on the solar disk. It is evident that many questions remain in the interpretation of the OSO-1 data which may be answered by future experiments combining spatial resolution with the existing spectral resolution.

\section{FLARE ACTIVITY OBSERVED BY OSO-1.}

The OSO-1 spectrometer observed transients events superimposed on the slow variations in the spectrum. These events could in most instances be correlated with other evidence of solar activity. It is difficult to obtain reliable data for such events because the increase in radiation is observed as only a small increase in the radiation from the entire disk, and because the long time required to scan the spectrum $(8 \mathrm{~min}$ ) severely limits the time resolution with which the event can be observed in a particular spectral line. Several tentative conclusions can be drawn from the data, however. In the first place, of all the EUV lines examined, the largest increase during flares is associated with the ion having the highest ionization potential Fe XVI. Secondly, even for this spectral line the increase is considerably less than for harder $\mathrm{X}$ rays. For a flare of importance $2^{+}$, on $13 \mathrm{March}$ 1962, the Fe XVI line at $\lambda 335$ increased by $60 \%$ whereas the 1-10 $\AA$ ion chamber output [17] increased by a factor of more than three.

Numerous transient events are indicated in Figure 9. In general the Fe XVI flux increases more than does $\mathrm{Fe} X V$, thus increasing the ratio $\lambda 335 / \lambda 284$. In many cases no change whatever in the Fe XV flux is observed during the flare. Figure 10 gives one example of a transient event.

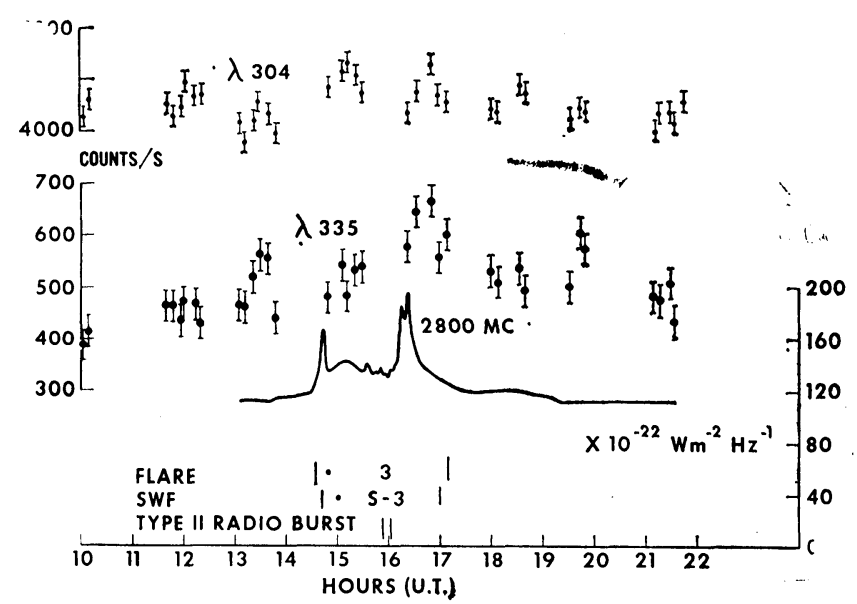

Fra. 10. - EUV Flare observation and comparison with a microwave burst observed by Covington (National Research Council, Ottawa, Canada). April 22, 1962.

The $\lambda 304$ line shows an increase of about $10 \%$ approximately coincident with the optical flare. A $30 \%$ is observed in the $\lambda \mathbf{3 3 5}$ line, but only after a type II radio burst has occurred. No change is observed in the $\lambda 284$ line. The observations are also compared with the radio event at $2800 \mathrm{MHz}$ reported by Covington of the National Research Council, Canada. Additional events, many of them indicated by increases in the Fe XVI/Fe XV ratio, are now being studied in greater detail.

\section{Conclusions.}

The solar extreme ultraviolet spectrum has been observed over a sufficiently long period of time to record emissions corresponding to varying levels of solar activity. All spectral lines are observed to vary, with those from ions which exist at high electron temperatures showing the greatest fluc- 
tuations. These variations have been applied to the problem of identifying unknown emission lines in the spectrum. The measurements have been compared with radio emission and other observa- tions of solar activity. It is concluded that EUV emission lines associated with an active region may not all have the same dependence on the age of the region.

\section{REFERENCES}

[1] Behring W. E., Neupert W. M., and LindSAY J. C., Space Research, III, ed. PrIEsTER W. (Amsterdam, North-Holland Publ. Co., 1963) 814.

[2] Neupert W. M., Behring W. E., and LindSAX J. C., Space Research, IV, ed. MULler P. (Amsterdam, North-Holland Publ. Co., 1964) 719 .

[3] Lindsay J. C., 1964, Planetary and Space Science $12,379$.

[4] Behring W. E., Neupert W. M., and NiCHoLs W. A., 1962, J. Opt. Soc. Am. 52 (5) : 597 May.

[5] Hall L. A., Damon K. R., and HinteregGER H. E., Space Research, III, ed. PrIEsTER W. (Amsterdam, North-Holland Publ. Co., 1963) 745 .

[6] Hinteregger H. E., Hall L. A., and SchweiZER W., 1964, $A p . J .140,319$.

[7] Tousey R., Austin W. E., Purcell J. D., and Widing K. G., Space Research, III ed. PriesTER W. (Amsterdam, North-Holland Publ. Co., 1963) 772.
[8] Zirin H., Hall L. A., and Hinteregger H. E. Space Research, III ed. Priester W. (Amsterdam, North-Holland Publ. Co., 1963) 760.

[9] Peck er C. and Rohrlich F., 1963, Ap. J. Suppl. No. 79.

[10] NeUpert W. M. and BeHring W. E., 1962 , J. Quant. Spectrosc. Radiat. Transfer 2, 527.

[11] Neupert W. M. and Smith E. V. P., Astron. $J$. (Abstract) to be published.

[12] Garstang R. H., 1962, Ann. d'Ap. 25, 109.

[13] Fawcett B. C., Gabriel A. H. Griffin W. G., Jones B. B., and Wilson R., 1963, Nature (GB) 200, 1303.

[14] ZIRIN H., (submitted for publication in $A p . \quad J$ ).

[15] House L. L., 1964, Ap. J. Suppl. No. 81.

[16] DE JAGER C., 1961. The Development of a Solar Centre of Activity, Vistas in Astronomy, Vol. 4 143.

[17] White W. A., Space Research, IV ed., MULLER P. (Amsterdam North-Holland Publ. Co., 1964) 771. 